RESEARCH PAPER

\title{
The traditional belief system in relation to mental health and psychiatric services in Sudan
}

\author{
E. A. Sorketti, N. Z. Zuraida and M. H. Habil
}

Department of Psychological Medicine, Faculty of Medicine, University of Malaya, Kuala Lumpur, Malaysia, email ehabsorketti@yahoo.com

The authors express their gratitude to all those with mental disorders in the traditional healers' centres and their families and relatives who answered our research questions. They were our essential guides and teachers for the better understan teachers for the better understanding of traditional healing and menta illness. We would also like to thank the traditional healers and their assisting therapists for welcoming the research teams.
Traditional healers' centres may constitute community resources for people with a mental illness. Many low-income countries are seeking to integrate mental health into their mainstream health services and primary healthcare, so as to decrease the duration of untreated illness. Traditional healers can help to meet these needs. A series of four studies has been conducted in central Sudan. In-patients with mental disorders undergoing treatment with traditional healers were recruited, as well as some of the healers themselves. The resulting observations should help practitioners trained in Western psychiatry to better understand traditional healing as an alternative healthcare system. The results should contribute to current debates on whether or not traditional healers in Africa should be officially recognised as healthcare providers. They should also deepen social scientists' understanding of the role of culture in mental health and help policy makers to improve mental health services.

Traditional healers' centres may constitute community resources for people with a mental illness in a culture where they are recognised and valued. Traditional healers often have high credibility and deep respect among the population. They are knowledgeable about local treatment options, as well as about the physical, emotional and spiritual lives of the people they serve, and are able to influence their behaviour. Therefore, it is imperative to consider traditional healers as partners in an expanded response to mental disorder, and to maximise the contribution they can make in meeting the needs of those who require some form of mental health service (Anderson \& Kaleeba, 2002, p. 5).

A series of four studies has been conducted in central Sudan (see Sorketti, 2008, 2009; Sorketti \& Habil, 2009; Sorketti et al, 2010; Sorketti et al, 2011), with the following aims:

- to delineate the sociodemographic characteristics of people with mental disorders who seek treatment from traditional healers

- to record their clinical presentations and diagnoses

- to establish the outcomes afforded by traditional approaches to the treatment of people with psychosis

- to generate a profile of traditional healers
- to investigate the knowledge, beliefs, attitudes and practices of the wider Sudanese community in relation to people with mental disorders, traditional healing and formal psychiatric services.

The resulting observations should help practitioners trained in Western psychiatry to better understand traditional healing as an alternative healthcare system, one that is used by a large section of the Sudanese population - as is the case in other African countries, and elsewhere. The results should contribute to current debates on whether or not traditional healers in Africa should be officially recognised as healthcare providers. They should also deepen social scientists' understanding of the role of culture in mental health. The data may help policy makers to improve mental health services.

\section{Method}

We used both qualitative and quantitative research methods for the four studies, which were conducted in selected traditional healers' centres in central Sudan. In-patients with mental disorders undergoing treatment in these centres and the traditional healers themselves were recruited. The sample size was calculated using the Kish-Leslie formula for a descriptive study.

Both qualitative and quantitative research methods were used. These included focus group discussion, in-depth interviews with key informants and healers, structured questionnaires (for interviews with both patients and traditional healers), the Mini International Neuropsychiatric Interview (MINI, to elicit the diagnosis) and the Positive and Negative Syndrome Scale (PANSS, to assess those with a psychosis, at both admission and discharge from the centres).

Ethical approval was obtained before the start of the study and informed consent was obtained from all participants.

\section{Results}

We interviewed more than 400 patients receiving treatment at traditional healers' centres and were able to follow-up 129 patients with psychotic disorders from admission until discharge from the centres, to study the outcomes of the interventions.

We were able to interview 28 traditional healers to assess their concepts, attitudes and practices in relation to mental disorder.

Focus group discussions were held with the relatives and families of patients treated in these centres. 


\section{Discussion}

We need to modify community concepts, attitudes and practices concerning mental health and the care of people who have a mental illness, to raise public awareness and to decrease the stigma of mental illness and enhance utilisation of services.

It is vital to establish channels of collaboration and common understanding between traditional healers and mental health professionals in those countries where the majority of people with mental illness consult traditional healers first. Traditional healers are in a position to help in the early detection of mental illness; in turn, early management will lead to better outcomes. Collaboration with psychiatrists will help to eliminate some potentially harmful methods of practice by the traditional healers, such as misdiagnosis, isolating patients in an unhealthy, non-hygienic environment, depriving patients of nutritional food, and beating patients. Collaboration can help to improve community awareness and decrease the stigma of mental illness. Use should be made of traditional healers' centres as community rehabilitation facilities for people with mental illness.

If the education of traditional healers can be improved, they may gain a better understanding of mental illness and the benefits of modern medication. This could be achieved through seminars, programmes and workshops to raise awareness of new psychiatric treatments.

It was evident from the studies that traditional healing can produce some improvement in the signs and symptoms of patients, even those with psychotic disorders, but, despite this, the approaches used by traditional healers do raise ethical and human rights issues, which need to be addressed.

We need to ensure community involvement in both the delivery and the utilisation of mental health services. Many low-income countries are seeking to integrate mental health services into mainstream general health services and primary healthcare, so as to decrease the duration of untreated mental illness, through early community detection. Collaboration with traditional healers would be of enormous strategic benefit in this regard.

More research is needed into traditional healing and mental health in Sudan, especially community needs and demands; to this end, the establishment of a specialist research institute for mental health and traditional healing in low- and middle-income countries would be of great advantage.

Although the researchers did their best to make the studies as comprehensive as possible, there were of course some limitations.

- Some harmful and even abusive practices are used by traditional healers in these centres, such as depriving patients of food. The researchers were only observers but were able to advise patients and their families to think about modern psychiatric treatment, and provided addresses of local mental health services.
- Psychiatrists' opinions about traditional healing practices and collaboration were not ascertained.

- For patients with a psychosis, consent to participate was obtained from a close relative.

- We were prohibited from taking photographs and making tape recordings.

- Transportation was often a difficulty for the research team because many of the centres were in remote villages.

We need to bring what was happening in these centres to the attention of our psychiatric colleagues and indeed of mental health professionals more widely, as well as to the attention of mental health service providers, decision-makers in the Ministry of Health, government officials and human rights organisations. Collaboration will help to improve the situation and put an end to some of the harmful practices we found. The current situation is most probably due to the shortage and high cost of formal mental health services, but also to the long experience in Sudan of war, internal conflict, political instability, poverty and lack of education.

Much work has to be done in order to convince patients' families and to work closely with the traditional healers to educate them (not to fight them) about mental illness and the value and effects of modern psychiatric treatment. There is at present a gap between psychiatrists, mental health professionals and people with mental disorders. This is true of many low-income countries. Psychiatrists and other service providers need to make more effort to reach those patients who require modern psychiatric management.

There is a lack of appropriate legislation to regulate traditional healers in Sudan (as in many other countries). Such legislation is urgently needed.

While it is important to know what role cultural and social factors play in determining the health-seeking behaviours of people with mental disorders and their relatives, it is imperative that researchers investigate what is going on in these centres from a cultural point of view, and that they do not judge them.

\section{References}

Anderson, S. \& Kaleeba, N. (2002) Ancient Remedies, New Disease: Involving Traditional Healers in Increasing Access to AIDS Care and Prevention in East Africa. UNAIDS.

Sorketti, E. A. (2008) General overview of traditional healer practices in relation to mental health in Sudan. Arabpsynet e.Journal, 18/19, 245-248.

Sorketti, E. A. (2009) Sudan's national mental health programme and burden of mental illness. International Psychiatry, 6, 16-18.

Sorketti, E. A. \& Habil, M. H. (2009) The current situation of the people with mental illness in the traditional healer centers in Sudan. Malaysian Journal of Psychiatry, 18, 78-81.

Sorketti, E. A., Zuraida, N. Z. \& Habil, M. H. (2010) Collaboration between traditional healers and psychiatrists in Sudan. International Psychiatry, 7, 71-74.

Sorketti, E. A., Zainal, N. Z. \& Habil, M. H. (2011) The characteristics of people with mental illness who are under treatment in traditional healer centres in Sudan. International Journal of Social Psychiatry, doi 10.1177/0020764010390439. 